\title{
Stellar orbital properties as diagnostics of the origin of the stellar halo
}

\author{
Monica Valluri ${ }^{1}$, Sarah R. Loebman ${ }^{1,2}$, Jeremy Bailin ${ }^{3}$, \\ Adam Clarke ${ }^{4}$, Victor P. Debattista ${ }^{4}$, Greg Stinson $^{5}$ \\ ${ }^{1}$ University of Michigan, USA email: mvalluri@umich.edu, ${ }^{2}$ Michigan Society of Fellows, \\ ${ }^{3}$ University of Alabama, USA, ${ }^{4}$ University of Central Lancashire, UK, ${ }^{5}$ Max Planck Institute \\ for Astronomie, Germany
}

\begin{abstract}
We examine metallicities, ages and orbital properties of halo stars in a Milky-Way like disk galaxy formed in the cosmological hydrodynamical MaGICC simulations. Halo stars were either accreted from satellites or they formed in situ in the disk or bulge of the galaxy and were then kicked up into the halo ("in situ/ kicked-up" stars). Regardless of where they formed both types show surprisingly similar orbital properties: the majority of both types are on short-axis tubes with the same sense of rotation as the disk - implying that a large fraction of satellites are accreted onto the halo with the same sense of angular momentum as the disk.
\end{abstract}

Keywords. Galaxy: halo, Galaxy: kinematics and dynamics, Galaxy: stellar content

\section{Introduction}

The orbital properties of halo stars from the MaGICC simulated galaxy g15784 - a realistic Milky Way sized disk galaxy at $z=0$ (Stinson et al. 2012) - are used to assess whether it is possible to determine the birth site of halo stars based on individual orbital properties. The dark matter halo of this galaxy is mildly triaxial at all radii. The stars were picked to lie within $100 \mathrm{kpc}$ of the center of the galaxy but not within the disk (i.e at $|z|>3 \mathrm{kpc}$ and $R>25 \mathrm{kpc}$ ). The formation sites of halo stars were determined by examining 100 snapshots of the simulation. "Accreted halo stars" form in satellites outside the virial radius and are subsequently added to the halo via tidal stripping. Stars that form "in-situ" in the main disk or bulge and are subsequently kicked into the halo are referred to as "in situ/ kicked-up" stars. The phase space coordinates of individual stars at $z=0$ were used to numerically integrate individual orbits in the frozen potential corresponding to the full galaxy potential. Frequency analysis of the orbits was used to classify orbits (Valluri et al. 2010, 2012) into the major orbit families found in triaxial halos: box orbits, short axis tubes (SAT), long axis tubes (LAT) and chaotic orbits.

\section{Orbits of accreted and "kicked-up" halo stars}

Orbits of 14,000 accreted stars 14,000 "kicked-up" stars were classified. Table 1 shows the percentage of accreted and "kicked-up" stars belonging to each orbit family. The majority of orbits are on SATs with the same sense of rotation as the disk, regardless of where they formed. This is probably because satellies are often accreted together along the same large-scale filaments that contribute to the hierarchical growth of the galaxy (Helmi et al. 2011). More surprising is the fact that the fractions of box orbits and chaotic orbits are independent of formation site: however these orbit families are more centrally concentrated in the halo and are likely to have experienced more chaotic scattering (Valluri et al. 2013). We find that the distribution of orbital chaoticity in this high resolution simulation is identical to that in controlled simulations (Valluri et al. 2010). 

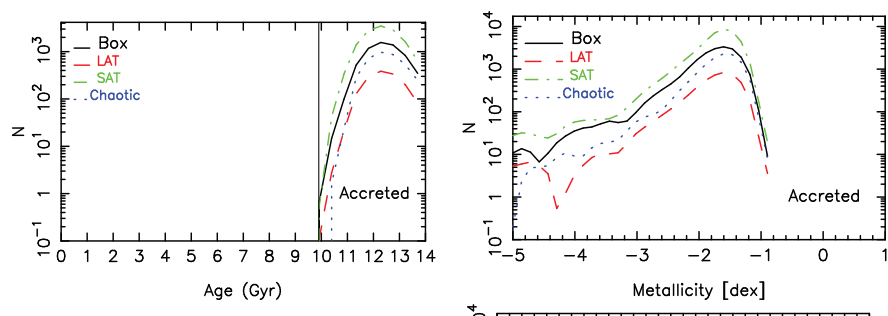

Table 1. Orbital Populations of Accreted vs. "Kicked up" halo stars
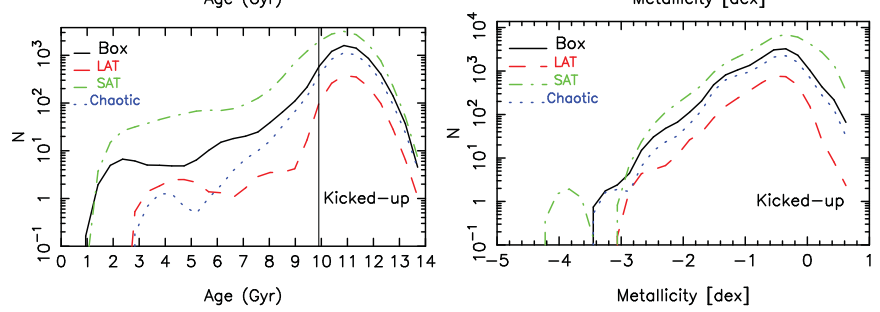

\begin{tabular}{lcc}
\hline Type & Accreted & "Kicked-up" \\
\hline Boxes & $27 \%$ & $25 \%$ \\
SAT & $57 \%$ & $59 \%$ \\
LAT & $6 \%$ & $6 \%$ \\
Chaotic & $10 \%$ & $10 \%$ \\
\hline
\end{tabular}

Figure 1. Kernel density histograms showing number of orbits of each family as a function of stellar age (left) and metallicity (right) for accreted stars (top row) and "in situ/ kicked up" stars (bottom row). Orbits of all families are similarly distributed in each panel. The thin vertical lines (at 9.9 Gyr) marks the youngest accreted stars.

Figure 1 shows that "accreted" and "in situ" stars have slightly different distributions with age and metalliticy, but all orbit families in a given panel show similar distrbutions in these quantities. Accreted stars are older on average than "in situ" stars but the peak of the "in situ" stars is also old. Likewise there is significant overlap in their metallicity distributions. Snaith et al. (2015) show that while the accreted halo stars have higher $\alpha$-abundances on average than "in situ" halo stars, there is overlap in this space too.

\section{Conclusions}

Orbital properties of halo stars are independent of whether they were accreted or whether they were formed in the disk/bulge and then "kicked-up" into the halo. Both types of stars are mainly on short-axis tubes $(\sim 60 \%)$, probably because the accretion of satellites occurs on a few large scale filaments that also feed the disk. Chaotic scattering by the central bulge put stars in the inner halo on box or chaotic orbits (Valluri et al. 2010). In this MaGICC galaxy the overlap in the ages, metallicities and orbital properties of halo stars makes it difficult to uniquely identify exactly where they formed. This analysis is being repeated for other disks to assess the dependence of this result on accretion history.

\section{Acknowledgements}

MV is supported by NASA-ATP grant NNX15AK79G. SRL is supported by the Michigan Society of Fellows, VPD is supported by STFC Consolidated grant \# ST/M000877/1. JB acknowledges support from HST-AR-12837, provided by NASA through a grant from the Space Telescope Science Institute.

\section{References}

Helmi, A. et al. 2011, ApJ 733, L7

Snaith, O. N. et al. MNRAS, submitted

Stinson, G. S., et al. 2012, MNRAS, 3506

Valluri, M., Debattista, V. P. et al. 2010, MNRAS, 403, 525

Valluri, M., Debattista, V. P. et al. 2012, MNRAS, 419, 1951

Valluri, M., Debattista, V. P., Stinson, G. S., Bailin, J. et al. 2013, Ap J., 767, 93 